\title{
Optical Retardation and Dispersion of Developable Domains in Columnar Hexagonal Liquid Crystal
}

Sattela $\mathbf{H}^{*}$, Loubiri I and Gharbia $M$

Faculty of Sciences of Tunis, Laboratory of Liquid Physics and Nonlinear Optics, Tunisia

\begin{abstract}
It's well known that the optical retardation measurement depends directly on the birefringence of the liquid crystal. We are interested by the simplest distorted texture of discotic columnar liquid crystals (DCLC) where the columns curve circularly their structures around the core line. The present opportunity is to use a hexagonal columnar structure where the topology of columns hemispheres is well known. We have observed the DCLC interference fringes from a half drop in the hexa-n-hexyloxytriphenylene close to the isotropic transition. Using polychromatic spectral lamp, the optical retardation symmetry has been used to describe the spherical texture of the columns. The present work shows that the optical retardation exhibits a noticeable difference between the birefringence measurement $\Delta \mathrm{n}$ versus the wavelength $\lambda$. The birefringence is given by the relationship: $\Delta n=\Delta n_{0}+B / \lambda 2,\left(\Delta n_{0}=-0,196\right.$ and $\left.B=0,023 \mu m^{2}\right)$.
\end{abstract}

Keywords: Birefringence; Columnar liquid crystal; Developable domains; Optical retardation; Dispersion

\section{Introduction}

Discotic Columnar Liquid Crystals (DCLC) are formed by a regular packing of parallel columns of disk like molecules; the columns are arranged in a two dimensional network [1-5]. For DCLC distortions, one has predicted [2] two types of elasticity curvature flexion for the columns and solid like elasticity for the 2D hexagonal crystal. Mechanical instability experiments have been made to test this model comparing curvature and compression $[3,6]$. This study helps to characterize the evolution of the columns topology in undulated developable domains [6-8] these domains growth in general around a core of typical radius $r_{c} \approx 1 \mu \mathrm{m}$.

We present the measurement of the bulk birefringence of DCLC developable domains through the observation of the variable thickness and molecular bend localized interference fringes in such a texture [9]. The hope of this work is to characterize optically the growth of a columnar half drop from the isotropic liquid phase taking into account the dimension of the core $r_{c}$ and growths in developable domains columns. Assume there is a DCLC sample in between two parallel glass plates $L_{1}$ and $L_{2}$. Since the isotropic phase, a slow decrease in temperature shows the usual sequence of Figures 1a-1c. In the textures of (Figures 1a and 1b), the topology is exactly known. The semi spherical symmetry of the Figure 2 retains the curvature of the columns and provides that the curved columns lead to a new line $\mathrm{S}_{1 / 2}$ carried by the $\mathrm{Oz}$ axis radius $\mathrm{r}_{\mathrm{c}}$ and probably filled with hexagonal phase [10]. In this geometry, we are interested in the shape of the half drop from centre to edge.



Figure 1: Optical microscopic pictures of circular developable domains (a,b), optical microscopic picture of germs with six dendrites characteristic of the hexagonal phase (c). Scale $13 \mu \mathrm{m}$ per division.
The new developable domains opportunity is related, in particular, to the observed textures of some developable domains types $\left(\mathrm{S}_{1 / 2}\right)$. Characterization of the texture will be made using the optical interference in the texture of the semi-circular half drop [11,12] (Figure 2).

\section{Experimental Setup}

In our experimental setup (Figure 3), an incident parallel light beam (white beam or a polychromatic beam) illuminates the sample with vertical polarization. We transform it into a circular polarization with a $\frac{\lambda}{4}$ plate. We selected the incident polarization with a linear polarizer. A webcam records the change in the sample texture. The obtained images are captured and transferred to the computer. The camera objective is fixed at the microscope ocular coaxially with the ocular.



Figure 2: Texture model and sample geometry. The lines represent the columns. The core is a line along $\mathrm{Oz}$. The outer volume of the columnar phase is limited by a circular profile.

*Corresponding author: Sattela H, Faculty of Sciences of Tunis, Laboratory of Liquid Physics and Nonlinear Optics, 1060, Belvedere Tunis, Tunisia, Tel:+216 71 882 200; E-mail: sattelahanen91@gmail.com

Received October 20, 2017; Accepted October 25, 2017; Published October 31, 2017

Citation: Sattela H, Loubiri I, Gharbia M (2017) Optical Retardation and Dispersion of Developable Domains in Columnar Hexagonal Liquid Crystal. J Laser Opt Photonics 4: 169. doi: 10.4172/2469-410X.1000169

Copyright: (C) 2017 Sattela H, et al. This is an open-access article distributed under the terms of the Creative Commons Attribution License, which permits unrestricted use, distribution, and reproduction in any medium, provided the original author and source are credited. 


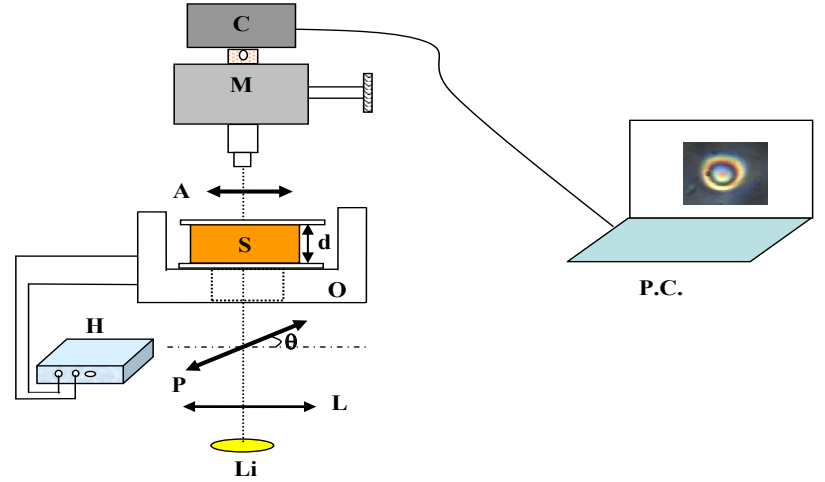

Figure 3: Experimental setup ( $\mathrm{Li}$ : incident white light or spectral lamp, $\mathrm{P}$ : polarizer, A: analyzer, $\mathrm{S}$ : sample, d: sample thickness, $M$ : microscope, O: Oven, $\mathrm{C}$ : camera, $\mathrm{H}$ : heater and $\mathrm{L}$ : lens).

The experimental setup comprises an upper plate $\mathrm{L}_{1}$ to a temperature $\mathrm{T}_{1}$ and a lower plate $\mathrm{L}_{2}$ to a temperature $\mathrm{T}_{2}$ with the possibility to create a thermal gradient controllable by means of appropriate regulation $\left(\mathrm{T}_{1}<\mathrm{T}_{2}\right)$.

In this experiment we hope making the measured temperature more precise and try to attend $0.02 \%$ of accuracy. To achieve our hope, we used a Kalman filter. A technique that allows us to eliminate the additive noises that deteriorate our measurements.

Before using the Kalman filter we draw the variation of temperature between $94^{\circ} \mathrm{C}$ and $95.5^{\circ} \mathrm{C}$. The obtained experimental results show that the accuracy of the measurement is $0.1^{\circ} \mathrm{C}$. After using the Kalman filter, the variation of the measured temperature is softer and the accuracy reaches $0.02^{\circ} \mathrm{C}$.

From the structure of Figure 2, the sample in isotropic phase on the plate $\mathrm{L}_{2}$ and in a liquid crystal phase on the plate $\mathrm{L}_{1}$, undergoes a slow decrease in temperature which shows whose radius can reach a few tens of $\mu \mathrm{m}$.

A decrease temperature $\mathrm{T}_{1}$ in $\mathrm{L}_{1}$ shows that the diameter of the half drop increases and progresses to the dendrites characteristic of the hexagonal phase (Figure 1c). Experimentally, to avoid the presence of dendrites and fix the semicircular structure, it is necessary to maintain the plate $\mathrm{L}_{2}$ at the temperature transition isotropic liquid-DCLC.

We illuminate the structure of the controlled half drop by an incident parallel light beam in order to characterize the optical retardation in the half drop. The thickness of the sample can be adjusted with mica spacers, from 6 to $80 \mu \mathrm{m}$. We fix the thickness to $\mathrm{d}=80 \mu \mathrm{m}$.

The sample used is the hexa-n-hexyloxytriphenylene $\left(\mathrm{C}_{6} \mathrm{HET}\right)$ which exhibits a discotic columnar mesophase between $69^{\circ} \mathrm{C}$ and $96^{\circ} \mathrm{C}$ [13]. The $\mathrm{L}_{1}$ temperature is fixed at $96^{\circ} \mathrm{C}$ and the $\mathrm{L}_{2}$ temperature decreases slowly from $96^{\circ} \mathrm{C}$. We can move the incident polychromatic beam away from the obtained semicircular structure.

\section{Experimental Results}

\section{Interference of developable domains in white light}

Calling $(\mathrm{x}, \mathrm{z})$ the transverse axes, $\theta$ the polar angle and $\mathrm{R}$ the radius of the columns. A developable domains section $(\mathrm{x}, \mathrm{z})$ shows a semicircular basis (Figure 2).

This texture has symmetry of revolution around the axe $\mathrm{Oz}$ with a radius of the core $r_{c}$. Optically, the texture has locally a negative uniaxial material around the director line. The light polarization perpendicular to the section $(\mathrm{x} ; \mathrm{z})$ sees always the ordinary index. The perpendicular polarization sees the extraordinary index $n^{\prime}$ which depends on the local orientation of the columns compared to the light rays. We predict, for this geometry, the circular symmetry of a standard interference fringes between ordinary and extraordinary rays.

In our experiment, the geometry is exactly known and in particular the extraordinary ray must be tilted by such a non-flat profile and should become a curved line in the variable index medium. To compute the optical path difference between ordinary and extraordinary rays, we assume that all light rays remain perpendicular to the plates. As already explained, we assume the columns to be semi circles centred on the core, we can write the classical expression of the local extraordinary index n' as:

$$
\frac{1}{\mathrm{n}_{\mathrm{e}}{ }^{2}}=\frac{\sin ^{2} \theta}{\mathrm{n}_{\mathrm{o}}{ }^{2}}+\frac{\cos ^{2} \theta}{\mathrm{n}_{\mathrm{e}}{ }^{2}}
$$

We deduce the optical retardation $\delta$ as :

$$
\delta(\mathrm{x})=\int_{0}^{\mathrm{zmax}}\left(\mathrm{n}_{\mathrm{e}}^{\prime}-\mathrm{n}_{\mathrm{o}}\right) \mathrm{dz}
$$

In the case of weak birefringence $\Delta \mathrm{n}=\mathrm{n}_{\mathrm{e}}-\mathrm{n}_{0}<<\mathrm{n}_{0}$. the expression (2) gives for each value $\theta_{0}$ of $\theta$ close to $\frac{\pi}{2}$ [14].

$$
\delta(\theta)=\mathrm{R} \Delta \mathrm{n}(\sin \theta-\theta \cos \theta)
$$

In all cases, the edge contains a black ring that identifies the radius $\mathrm{R}$ of the half drop.

This optical retardation expression (3) depends only on the polar angle $\theta$ and predicts a circular symmetry. Close to the core $\left(\frac{x 0}{R}<<1\right)$ and for small values of $\mathrm{R}$, one can estimate the optical retardation, using a reasonable value of $\Delta \mathrm{n} \approx-0.14$ [1] as $\mathrm{R} \Delta \mathrm{n}=-4.2 \mu \mathrm{m}$. This low value shows that, the halfdrop willpresent theNewton tints, the order of fringes increases from the edge $(x=R$ and $\delta=0)$ to the center $(x=0$ and $\delta=\mathrm{R} . \Delta \mathrm{n})$.

The radius $\mathrm{R}$ of the half drop is limited to a few tens of microns ( $\mathrm{R}$ $\leq 30 \mu \mathrm{m})$. This radiusis less than the thickness $\mathrm{d}=80 \mu \mathrm{m}$ which shows that the drop is only half surrounded byisotropic liquid.

Between polarizer and analyzer perpendicul $(\mathrm{P} \perp \mathrm{A})$ we observe the existence of Newton tints provided by eqn. (3) which achievesthe interpretation of the fringes in rings with rotational symmetry around the axis $\mathrm{Oz}$ (Figure 4a). This symmetry is maintained even if the half drop evolves into aslightly elliptical shape (Figure $4 \mathrm{~b}$ ). The maximum optical retardation precision is obtained for the central fringe: $\delta_{0}=\mathrm{R} \Delta \mathrm{n}=\mathrm{R}\left(\mathrm{n}_{\mathrm{e}}-\mathrm{n}_{0}\right)$. In all cases, the edge contains a black ring that identifies the half drop radius $\mathrm{R}$. By rotating the analyzer of an angle $\frac{\pi}{2}(\mathrm{p} \| \mathrm{A})$ we verify the existence of complementary colors also in rings where the optical retardation is given by the complementary Newton tints.

\section{Interference of developable domains in polychromatic light}

We illuminate the center of the rings by aspectral lamp of cadmium (Cd) which formed of four monochromatic radiations $\left(\lambda_{1}, \lambda_{2}, \lambda_{3}\right.$ and $\left.\lambda_{4}\right)$. The use of polychromatic light gives interference rings thinner and more contrasted. The experience shows, in particular, the absence of yellow color and the presence of only three rings: blue, green and red. 

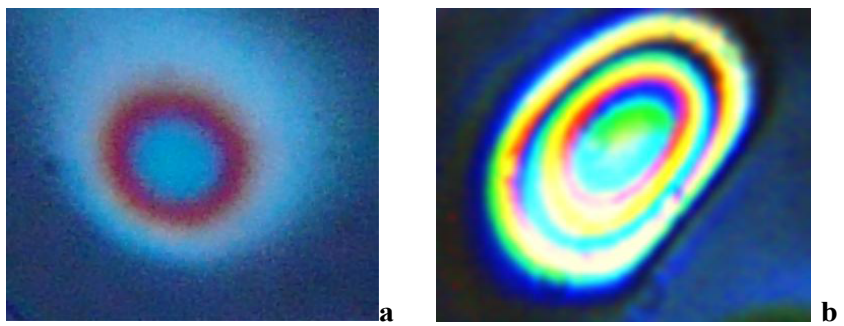

Figure 4: Experimental interference figures of developable domains seen under polarising microscope using incident white light beam. Scale $5 \mu \mathrm{m}$ per division.



Figure 5: Experimental interference picture of developable domain.

In practice, it is difficult to discernthe twoblue rings corresponding to the two wavelengths $\lambda_{1}$ (blue) and $\lambda_{2}$ (Indigo). We consider that the blue rings correspond to the two blue wavelength $\left(\lambda_{1}=465 \mathrm{~nm}\right.$ and $\lambda_{2}=480 \mathrm{~nm}$ ) with an average wavelength $\lambda_{1}^{\prime}=\frac{\lambda 1+\lambda 2}{2}=472 \mathrm{~nm}$. Using a webcam, we obtain a video film of the scrolling interference rings. We interpose on the video film projection successively band pass filter blue, green and red. Each filter selects monochromatic wavelengths $\lambda_{1}^{\prime} \lambda_{3}$ or $\lambda_{4}$ contained in the filter band pass. For example, the Figure 5 shows the experimental interference picture of developable domain.

Let's call the angle $\alpha=\frac{\pi}{2}-\theta$. By limiting the angular with that $\left(\alpha \leq 10^{\circ}\right.$, we can consider that $\alpha^{2}$ is negligible compared to 1 and that the optical retardation of the central spot $\delta(\alpha=0)=\mathrm{R} \Delta \mathrm{n}(\mathrm{a}$ few hundredths). The experiment involves heating a $\mathrm{C}_{6} \mathrm{HET}$ halfdrop of radius, $\mathrm{R}=30 \mu \mathrm{m}$ and observe the scrolling of the central ring if or the wavelength $\lambda_{\mathrm{i}}$.

For a chosen radius $\mathrm{R}$, the wavelength $\lambda_{\mathrm{i}}$ gives an illuminated central ring. The optical retardation, from the center to the first ring, is equal toone wavelength. Increasing $\mathrm{R}$, the interference figure shows scrolling rings from the center towards the outside. Inversely, when melting a half drop of radius $\mathrm{R}=30 \mu \mathrm{m}$, we observe the scrolling rings toward the center. In both cases, the central ringis even more resolved that $\mathrm{R}$ is large. For moreprecision, this requires selecting values of $\mathrm{R}$ greater than $10 \mu \mathrm{m}$ and diameter central ring of the order $4 \mu \mathrm{m}$.

\section{Measurement of the birefringence vs. the wavelength}

Quantitatively, we increase the radius of the halfdropand we measurethe optical retardation corresponding to the central ring.

The optical retardation between the $\mathrm{k}$ successive ringsscrollis $(\mathrm{k}-1)$ wavelengths. This experiment allows to increase the optical retardation $\delta=\mathrm{k} \lambda$ by increasing the radius R. Figure 6 shows the respective experimental curves $\delta$ versus $\mathrm{R}$ for the three wavelengths $\lambda^{\prime}, \lambda_{3}$ and $\lambda_{4}$. It's easy to see that plotting $\delta$ as a function of $R$, the slope of the line provides the measure of the birefringence. The comparison of the slopes relative to the three wavelengths leads us to suppose that the birefringence is greater when the wavelength increase. The qualitative measurements of $\Delta \mathrm{n}$ for each wavelength give respectively: $\Delta \mathrm{n}\left(\lambda_{1}^{\prime}\right)=-0.092$ $\pm 0.005, \Delta \mathrm{n}\left(\lambda_{3}\right)=-0.106 \pm 0.005$ and $\Delta \mathrm{n}\left(\lambda_{4}\right)=-0.140 \pm 0.005$. This study shows that the birefringence increases as the wavelength increases unlike the 5CB nematic phase [15]. Note that, the three curves of Figure 6 do not pass through the origin. The intercept is used to estimate value ofcore radiusr of theorder of $1 \mu \mathrm{m}$.

In conclusion, this experimental method allows the study of the light dispersion by the columnar $\mathrm{C}_{6} \mathrm{HET}$.

\section{Quantitative study of the dispersion and discussion}

For the quantitative study of light dispersion, we hold on Figure 7 the values of $\Delta \mathrm{n}$ versus $\frac{1}{\lambda^{2}}$. This curve shows a linear behavior with an equation: $\Delta \mathrm{n}=\Delta \mathrm{n}_{0}+\frac{\mathrm{B}}{\lambda^{2}}$, similar to the Cauchy relation with: $\Delta \mathrm{n}_{0}=-0.195$ \pm 0.005 and $\mathrm{B}=0.023 \pm 0.005$.

This dispersion measurements show that $\Delta \mathrm{n}$ decreases for smaller wavelength, probably because increasing of $n_{e}$ larger than $n_{o}[16]$.

Forthe central spot, the uncertainty is closeto a few hundredths.



Figure 6: Measured optical retardation $\delta$ versus the radius $\mathrm{R}$ for the three wavelengths $\lambda_{1}^{\prime}=472 \mathrm{~nm}(\mathrm{a}), \lambda_{3}=509 \mathrm{~nm}(\mathrm{~b})$, and $\lambda_{4}=644 \mathrm{~nm}(\mathrm{c})$.

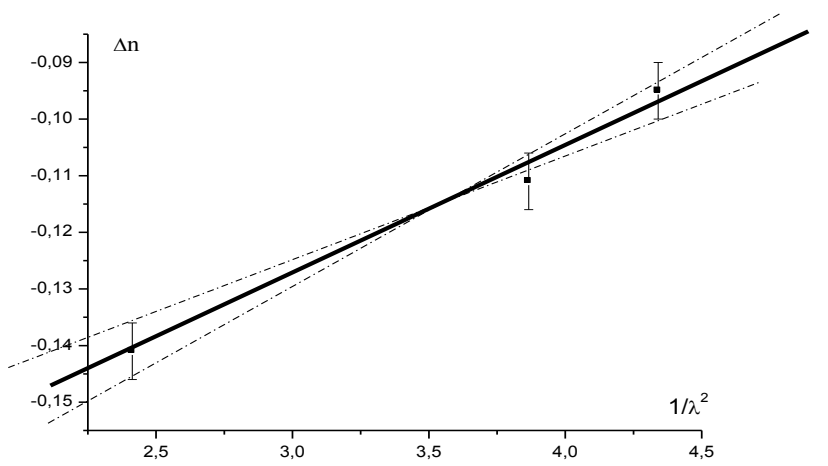

Figure 7: For the three wavelengths $\lambda_{\mathrm{i}}$ calculated, birefringence $\Delta \mathrm{n}_{\mathrm{i}}$ versus $1 / \lambda_{i}^{2}$. 
Citation: Sattela H, Loubiri I, Gharbia M (2017) Optical Retardation and Dispersion of Developable Domains in Columnar Hexagonal Liquid Crystal. J Laser Opt Photonics 4: 169. doi: 10.4172/2469-410X.1000169

Page 4 of 4

We can consider that the profile is flat, which has a negligible effect onthe value of the birefringence.

For the red wavelength $\left(\lambda_{4}\right)$, the measured value of the biref ringence is in good agreement with that found inreference; $\Delta \mathrm{n}=-0.141$.

We can deduce the extraordinary index valuen for $\mathrm{n}_{\mathrm{o}}$ constant $\left(n_{0}=1.540\right)$. The values of $n_{e}$ for the three wavelengths are: $1.448,1.434$ and 1.400 .

Using this method, we can fix the interference order that avoids the inaccuracy of the order found in other studies [17]. This method allows measuring the birefringence only in the vicinity of the transition temperature, in comparison with the monocrystalline structure in which the study of the birefringence as a function of temperature is possible [18].

The principle of this method is to deduce the birefringence for the wavelength $\lambda$ from the measurement of the radius of the half drop. It is obvious that this method would be impossible for CLC lyotropic where $\Delta \mathrm{n}$ is of the order $1,5.10^{-3}[19]$, because the radius of the half drop is of the order of $1 \mathrm{~mm}$.

\section{Summary}

We have performed a thermal control to realize temperatures precision of the order of $0.02^{\circ} \mathrm{C}$ and temperature gradients better than $0.002^{\circ} \mathrm{C}$ per minute. This regulation has setnews developable domains called $S_{1 / 2}^{\prime}$ halfdrops as characterized by the presence of a new line $S_{1 / 2}^{\prime}$ of radial core.

Experimentally, for a fixed radius $\mathrm{R}$ of the half drop, the optical retardation symmetry allows characterization of the interference figure.

In parallel incident white light, we have shownthe existence of interference fringes witch have a circular symmetry. Using a polychromatic incident spectral lamp beam, we note that, the rings are more determined and show a clear dispersion according to the wavelengths.

Using the precision of the central ring, we measured the optical retardation. This measure allows to characterize the dispersion of light, and gives the following values of the birefringence for the wavelengths of the cadmium spectral lamp: $\Delta \mathrm{n}\left(\lambda_{1}^{\prime}\right)=-0.092 \pm 0.005, \Delta \mathrm{n}\left(\lambda_{3}\right)=-0.106$ \pm 0.005 and $\Delta \mathrm{n}\left(\lambda_{4}\right)=-0.140 \pm 0.005$.

The birefringence obeys to the Cauchy relation. It allows to estimate, for $n_{\mathrm{o}}$ constant, the values of $\mathrm{n}_{\mathrm{e}}=1.448,1.434$ and 1.400 successively for the three wavelengths $\lambda_{1}^{\prime}, \lambda_{3}$ and $\lambda_{4}$ of cadmium spectral lamp [19].

\section{References}

1. Destrade C, Tinh NH, Gasparoux H, Malthete J, Levelut AM (1981) Disk-like mesogens: a classification. Mol Cryst Liq Cryst 71: 111-135.

2. Kleman M, Oswald P (1982) Columnar discotic mesophases: elasticity, dislocations, instabilities. J Phys 43: 655-662.

3. Gharbia M, Cagnon M, Durand (1982) Column undulation instability in a discotic liquid crystal. J Physique 43: 655-662.

4. Cagnon M, Gharbia M, Durand G (1984) Column buckling instability in a discotic liquid crystal. Phys Rev Lett 53: 938-940.

5. Raïs K, Daoud M, Gharbia M, Gharbi A, Nguyen HT (2001) Column correlation in polycatenar mesophases. Chem Phys Chem 2: 45-49.

6. Gharbia M, Othmen T, Gharbi A, Destrade C, Durand G (1992) Raleigh scattering from column undulations in a discotic liquid crystal. Phys Rev Lett 68: 2031-2034.

7. Loubiri, I, Sanaâ F, Gharbia M (2017) Refractive index and dispersion measurements in prismatic thin film of phasmidic columnar liquid crystals $\mathrm{Mol}$ Crystal Liq Cryst, pp: 22-28.

8. Sanaâ F, Yakoubi A, Gharbia M (2007) Characterisation of the columnar undulations using rayleigh scattering. AIP Conference Proceedings 935: 137-142.

9. Dumitraşcu L, Dumitraşcu I, Dorohoi DO, Mihai T (2008) Interferometric method for birefringence determination with a polarizing microscope. Opt Express 16 : 20884-20890.

10. Oswald $P$, Pieransky $P,(2006)$ Smectic and columnar liquid crystals, Taylor \& Francis Group, pp: 575-609.

11. Sanna F, Palieme JF, Yakoubi A, Gharbia M (2016) Opt Mater 57: 193-201.

12. Tinh NH, Dubois JS, Malthete J, Destrade C, (1978) The syntheses of hexaalcoxy triphényléne, new mesophase. CR Acad Sci Paris 286: 463.

13. Benkelfat BE, Horache EH, Qin Zou, Vinouze B (2003) An electro-optic modulation technique for direct and accurate measurement of birefringence. Opt Comm 221: 271-278.

14. Li J, Wu ST (2004) Two-coefficient Cauchy model for low birefringence liquid crystals. J Appl Phys 96: 170-174.

15. Diaconu I, Melniciuc-Puic N, Dorohoi D, Aflori M, (2007) Birefringence dispersion of $\mathrm{N}$-(4-methoxybenzylidene)-4-butylaniline (MBBA) determined from channelled spectra. Spectrochim Acta A Mol Biomol Spectrosc 68: 536-541.

16. Nagarajan K, Shashidharan Nair CK (2007) The use of fractional orders in the determination of birefringence of highly dispersive materials by the channelled spectrum method. Opt Comm 275: 348-353.

17. Li J, Gauza S, Wu ST (2004) Temperature effect on liquid crystal refractive indices. J Appl Phys 96: 11-24.

18. Sallen L, Sotta $P$, Oswald $P$, (1997) Pretransitional effects near the hexagonalmicellar phase transition of the $\mathrm{C} 12 \mathrm{EO} / \mathrm{H} 2 \mathrm{O}$ lyotropic mixture. J Phys Chem B 101: 4875-4881.

19. Sanna F, Palieme JF, Yakoubi A, Gharbia M (2016) Opt Mater 57: $193-201$. 